\title{
COUNTABLY INFINITELY MANY POSITIVE SOLUTIONS FOR EVEN ORDER BOUNDARY VALUE PROBLEMS WITH STURM-LIOUVILLE TYPE INTEGRAL BOUNDARY CONDITIONS ON TIME SCALES
}

\author{
K. R. PRASAD* AND MD. KHUDDUSH
}

\begin{abstract}
In this paper, we establish the existence of countably infinitely many positive solutions for a certain even order two-point boundary value problem with integral boundary conditions on time scales by using Hölder's inequality and Krasnoselskii's fixed point theorem for operators on a cone.
\end{abstract}

\section{INTRODUCTION}

The study of dynamic equations on time scales unifies existing results in differential and finite difference equations, and provides powerful new tools for exploring connections between the traditionally separated fields. For details refer to the books by Bohner and Peterson [6], [7], Lakshmikantham et al. [23] and the papers [1], [3], [19].

The boundary value problems with integral boundary conditions occur in the study of nonlocal phenomena in many different areas of applied mathematics, physics and engineering, in particular, in heat conduction, chemical engineering, underground waterflow, thermo-elasticity, plasma physics, [2], [10], [11], [21], [22], [27], [33], [36] and reference therin. Recently, authors established the existence of positive solutions to boundary value problems with integral boundary conditions on time scales; for details, see [9], [12], [13], [18], [26], [28], [32], [34] and reference therein.

However, to the best of our knowledge, little work has been done on the existence of positive solutions for higher order boundary value problems with integral boundary conditions on time scales. We would like to mention some results of Karasa and Tokmak [20], Li and Wang [25], Li and Sun [24], Cetin and Topal [8], and Sreedhar et al [31] which motivate us to consider the problem (1.6)-(1.7). In 2013, Karasa and Tokmak [20] established the existence of a positive solution of the following third order boundary value problem with integral boundary conditions,

$$
\left.\begin{array}{c}
\left(\phi\left(-u^{\Delta \Delta}(t)\right)\right)^{\Delta}+q(t) f\left(t, u(t), u^{\Delta}(t)\right)=0, t \in[0,1]_{T}, \\
\Delta(0)=\int_{0}^{1} g_{1}(s) u(s) \Delta s, c u(1)+d u^{\Delta}(1)=\int_{0}^{1} g_{2}(s) u(s) \Delta s, u^{\Delta \Delta}(1)=0
\end{array}\right\}
$$

by using four functionals fixed point theorem. In the same year, Li and Wang [25] studied the existence of a positive solution of the following nonlinear third order boundary value problem with integral boundary conditions,

$$
\left.\begin{array}{c}
\left(\phi\left(-u^{\Delta \Delta}(t)\right)\right)^{\Delta}+q(t) f\left(t, u(t), u^{\Delta}(t)\right)=0, t \in[0,1]_{T}, \\
a u(0)-b u^{\Delta}(0)=\int_{0}^{1} g_{1}(s, u(s)) \Delta s, c u(1)+d u^{\Delta}(1)=\int_{0}^{1} g_{2}(s) u(s) \Delta s, u^{\Delta \Delta}(1)=0,
\end{array}\right\}
$$

by applying a generalization of the Leggett-Williams fixed point theorem. In [24], Li and Sun studied the following boundary value problem on time scales,

$$
\left.\begin{array}{c}
x^{\Delta}(t)+p(t) x^{\sigma}(t)=f\left(t, x^{\sigma}(t)\right), t \in(0, T) \mathbb{T}, \\
x(0)-\beta x^{\sigma}(T)=\alpha \int_{0}^{\sigma(T)} x^{\sigma}(s) \Delta g(s),
\end{array}\right\}
$$

Received $14^{\text {th }}$ August, 2017; accepted $18^{\text {th }}$ October, 2017; published $1^{\text {st }}$ November, 2017.

2010 Mathematics Subject Classification. 34B18, 34N05.

Key words and phrases. Green's function; boundary value problem; positive solution; cone.

(C) 2017 Authors retain the copyrights of their papers, and all open access articles are distributed under the terms of the Creative Commons Attribution License. 
where $x^{\sigma}=x \circ \sigma$, and by using fixed point index theory the existence of infinitely many positive solutions for (1.3) are obtained. In [8], Cetin and Topal investigated the existence of solutions for integral boundary value problems on time scales,

$$
\left.\begin{array}{c}
{\left[p(t) x^{\Delta}(t)\right]^{\nabla}+q(t) x(t)=f(t, x(t)), t \in[a, b]_{T},} \\
\alpha x(\rho(a))-\beta x^{[\Delta]}(\rho(a))=\int_{\rho(a)}^{b} h_{1}(x(s)) \nabla s, \gamma x(b)+\delta x^{[\Delta]}(b)=\int_{\rho(a)}^{b} h_{2}(x(s)) \nabla s,
\end{array}\right\}
$$

by using Schauder fixed point theorem in a cone and by the method of upper and lower solutions. In 2017, Sreedhar et al [31] considered the $2 n^{\text {th }}$ order boundary value problem with integral boundary conditions on time scales,

$$
\left.\begin{array}{c}
(-1)^{n} u^{\Delta^{2 n}}(t)=f(t, u(t)), t \in(0,1)_{\mathbb{T}}, \\
u^{\Delta^{2 i}}(0)=u^{\Delta^{2 i}}(1)=\int_{0}^{1} a_{i+1}(x) u^{\Delta^{2 i}}(x) \Delta x, 0 \leq i \leq n-1,
\end{array}\right\}
$$

where $n$ act as positive. By using Avery-Henderson fixed point theorem, the authors established the existence of even number of positive solutions for (1.5).

Motivated by the work mentioned above, in this paper we investigate the existence of infinitely many positive solutions for the even order boundary value problem on time scales given by

$$
(-1)^{n} u^{(\Delta \nabla)^{n}}(t)=\omega(t) f(u(t)), t \in[0,1]_{\mathbb{T}},
$$

satisfying the Sturm-Liouville type integral boundary conditions

$$
\left.\begin{array}{c}
\alpha_{i+1} u^{(\Delta \nabla)^{i}}(0)-\beta_{i+1} u^{(\Delta \nabla)^{i} \Delta}(0)=\int_{0}^{1} a_{i+1}(s) u^{(\Delta \nabla)^{i}}(s) \nabla s, 0 \leq i \leq n-1, \\
\gamma_{i+1} u^{(\Delta \nabla)^{i}}(1)+\delta_{i+1} u^{(\Delta \nabla)^{i} \Delta}(1)=\int_{0}^{1} b_{i+1}(s) u^{(\Delta \nabla)^{i}}(s) \nabla s, 0 \leq i \leq n-1,
\end{array}\right\}
$$

where $n \geq 1, \mathbb{T}$ is a time scale, $f \in C([0,+\infty),[0,+\infty)), \omega(t) \in L_{\nabla}^{p}[0,1]$ for some $p \geq 1$ and has countably many singularities in $\left(0, \frac{1}{2}\right) \mathbb{T}$. We show that the boundary value problem (1.6)-(1.7) has countably infinitely many positive solutions by imposing suitable conditions on $\omega$ and $f$. The key tool in our approach is the Hölder's inequality and Krasnoselskii's fixed point theorem for operators on a cone.

\section{Preliminaries}

In this section, we provide some definitions and lemmas which are useful for our later discussions; for details, see [2], [4], [5], [6], [16], [30], [35].

Definition 2.1. A time scale $\mathbb{T}$ is a nonempty closed subset of the real numbers $\mathbb{R}$. $\mathbb{T}$ has the topology that it inherits from the real numbers with the standard topology. It follows that the jump operators $\sigma, \rho: \mathbb{T} \rightarrow \mathbb{T}$,

$$
\sigma(t)=\inf \{r \in \mathbb{T}: r>t\}, \quad \rho(t)=\sup \{r \in \mathbb{T}: r<t\}
$$

(supplemented by $\inf \emptyset:=\sup \mathbb{T}$ and $\sup \emptyset:=\inf \mathbb{T}$ ) are well defined. The point $t \in \mathbb{T}$ is left-dense, left-scattered, right-dense, right-scattered if $\rho(t)=t, \rho(t)<t, \sigma(t)=t, \sigma(t)<t$, respectively.

By an interval time scale, we mean the intersection of a real interval with a given time scale. i.e., $[a, b] \mathbb{T}=[a, b] \cap \mathbb{T}$ other intervals can be defined similarly.

Definition 2.2. Let $\mu_{\Delta}$ and $\mu_{\nabla}$ be the Lebesgue $\Delta$ - measure and the Lebesgue $\nabla$-measure on $\mathbb{T}$, respectively. If $A \subset \mathbb{T}$ satisfies $\mu_{\Delta}(A)=\mu_{\nabla}(A)$, then we call $A$ is measurable on $\mathbb{T}$, denoted $\mu(A)$ and this value is called the Lebesgue measure of $A$. Let $P$ denote a proposition with respect to $t \in \mathbb{T}$.

(i) If there exists $E_{1} \subset A$ with $\mu_{\Delta}\left(E_{1}\right)=0$ such that $P$ holds on $A \backslash E_{1}$, then $P$ is said to hold $\Delta$-a.e. on $A$.

(ii) If there exists $E_{2} \subset A$ with $\mu_{\nabla}\left(E_{2}\right)=0$ such that $P$ holds on $A \backslash E_{2}$, then $P$ is said to hold $\nabla$-a.e. on $A$. 
Definition 2.3. Let $E \subset \mathbb{T}$ be $a \nabla$-measurable set and $p \in \overline{\mathbb{R}} \equiv \mathbb{R} \cup\{-\infty,+\infty\}$ be such that $p \geq 1$ and let $f: E \rightarrow \overline{\mathbb{R}}$ be $\nabla$-measurable function. We say that $f$ belongs to $L_{\nabla}^{p}(E)$ provided that either

$$
\int_{E}|f|^{p}(s) \nabla s<\infty \quad \text { if } \quad p \in \mathbb{R}
$$

or there exists a constant $M \in \mathbb{R}$ such that

$$
|f| \leq M, \nabla-\text { a.e. on } E \text { if } p=+\infty \text {. }
$$

Lemma 2.1. Let $E \subset \mathbb{T}$ be a $\nabla$-measurable set. If $f: \mathbb{T} \rightarrow \mathbb{R}$ is a $\nabla$-integrable on $E$, then

$$
\int_{E} f(s) \nabla s=\int_{E} f(s) d s+\sum_{i \in I_{E}}\left(t_{i}-\rho\left(t_{i}\right)\right) f\left(t_{i}\right)
$$

where $I_{E}:=\left\{i \in I: t_{i} \in E\right\}$ and $\left\{t_{i}\right\}_{i \in I}, I \subset \mathbb{N}$, is the set of all left-scattered points of $\mathbb{T}$.

For convenience, we introduce the following notation throughout the paper: For $\tau \in\left(0, \frac{1}{2}\right) \mathbb{T}$,

$$
\begin{aligned}
& \phi_{i}(t):=\gamma_{i}+\delta_{i}-\gamma_{i} t, \psi_{i}(t):=\beta_{i}+\alpha_{i} t, d_{i}:=\gamma_{i} \beta_{i}+\alpha_{i} \delta_{i}+\alpha_{i} \gamma_{i}, \\
& G_{i}(t, s):=\frac{1}{d_{i}}\left\{\begin{array}{l}
\phi_{i}(s) \psi_{i}(t), t \leq s, \\
\phi_{i}(t) \psi_{i}(s), s \leq t,
\end{array} \quad A:=\int_{0}^{1}\left[\int_{0}^{1} G_{i}(s, r) a_{i}(s) \nabla s\right] \omega(r) \nabla r,\right. \\
& B:=\int_{0}^{1}\left[\int_{0}^{1} G_{i}(s, r) b_{i}(s) \nabla s\right] \omega(r) \nabla r, u_{i}:=\frac{1}{d_{i}} \int_{0}^{1} a_{i}(t) \phi_{i}(t) \nabla t, u_{i}^{*}:=\frac{1}{d_{i}} \int_{0}^{1} a_{i}(t) \psi_{i}(t) \nabla t, \\
& v_{i}:=\frac{1}{d_{i}} \int_{0}^{1} b_{i}(t) \psi_{i}(t) \nabla t, v_{i}^{*}:=\frac{1}{d_{i}} \int_{0}^{1} b_{i}(t) \phi_{i}(t) \nabla t, \\
& \theta_{i}(t):=\frac{\left(1-v_{i}\right) \phi_{i}(t)+v_{i}^{*} \psi_{i}(t)}{d_{i}\left(\left(1-u_{i}\right)\left(1-v_{i}\right)-u_{i}^{*} v_{i}^{*}\right)}, \zeta_{i}(t):=\frac{\left(1-u_{i}\right) \psi_{i}(t)+u_{i}^{*} \phi_{i}(t)}{d_{i}\left(\left(1-u_{i}\right)\left(1-v_{i}\right)-u_{i}^{*} v_{i}^{*}\right)}, \\
& \theta_{i}^{*}:=\max _{t \in J_{0}} \theta_{i}(t), \theta_{i}^{* *}:=\max _{t \in[\tau, 1-\tau]} \theta_{\mathbb{T}}(t), \zeta_{i}^{*}:=\max _{t \in J_{0}} \zeta_{i}(t), \zeta_{i}^{* *}:=\max _{t \in[\tau, 1-\tau]} \zeta_{\mathbb{T}}(t), \\
& a_{i}^{*}:=\int_{0}^{1} a_{i}(t) \nabla t, a_{i}(\tau):=\int_{\tau}^{1-\tau} a_{i}(t) \nabla t, b_{i}^{*}:=\int_{0}^{1} b_{i}(t) \nabla t, b_{i}(\tau):=\int_{\tau}^{1-\tau} b_{i}(t) \nabla t, \\
& g_{i}:=\int_{0}^{1} G_{i}(t, t) \nabla t, g_{i}(\tau):=\int_{\tau}^{1-\tau} G_{i}(t, t) \nabla t, \text { and } J_{0}:=[0,1]_{\mathbb{T}} .
\end{aligned}
$$

We make the following assumptions throughout the paper:

(H1) there exists a sequence $\left\{t_{k}\right\}_{k=1}^{\infty}(k \in \mathbb{N}), t_{1}<\frac{1}{2}, \lim _{k \rightarrow \infty} t_{k}=t^{*} \geq 0$ and $\lim _{t \rightarrow t_{k}} \omega(t)=+\infty$ for all $k=1,2,3, \cdots$.

(H2) $\omega \in L_{\nabla}^{p}\left(J_{0}\right)$ for some $1 \leq p \leq+\infty$ and there exists $m>0$ such that $\omega(t) \geq m$ for all $\left[t^{*}, 1-t^{*}\right]_{\mathbb{T}}$

(H3) $f: J_{0} \times[0,+\infty) \rightarrow[0,+\infty)$ is continuous,

(H4) $a_{i}, b_{i} \in L_{\nabla}^{1}\left(J_{0}\right)$ for all $1 \leq i \leq n$, are nonnegative and $\left(1-u_{i}\right)\left(1-v_{i}\right)-v_{i}^{*} u_{i}^{*}>0$ for all $1 \leq i \leq n$, on $J_{0}$,

(H5) $\alpha_{i}, \beta_{i}, \gamma_{i}, \delta_{i} \geq 0$ such that $d_{i}:=\gamma_{i} \beta_{i}+\alpha_{i} \delta_{i}+\alpha_{i} \gamma_{i}>0$ for each $1 \leq i \leq n$.

The rest of the paper is organized in the following fashion. In Section 2, we provide some definitions and lemmas that provide us with some useful information concerning the behavior of solution of the boundary value problem (1.6)-(1.7). In Section 3, we construct the Green's function for the homogeneous problem corresponding to (1.6)-(1.7), estimate bounds for the Green's function, and some lemmas which are needed in establishing our main results are provided. In Section 4, we establish a criteria for the existence of countable number of positive solutions for the boundary value problem (1.6)-(1.7) by applying Krasnoselskii's fixed point theorem in cones. Finally, we provide an example of a family of functions $\omega(t)$ that satisfy conditions $(H 1)-(H 3)$. 


\section{Green's Function And Bounds}

In this section, we construct the Green's function for the homogeneous problem corresponding to (1.6)-(1.7) and estimate bounds for the Green's function.

Lemma 3.1. Let $(H 4),(H 5)$ hold. Then for any $h(t) \in C\left(J_{0}\right)$, the boundary value problem,

$$
\begin{gathered}
-u^{\Delta \nabla}(t)=h(t), t \in J_{0}, \\
\alpha_{i} u(0)-\beta_{i} u^{\Delta}(0)=\int_{0}^{1} a_{i}(s) u(s) \nabla s, 1 \leq i \leq n, \\
\gamma_{i} u(1)+\delta_{i} u^{\Delta}(1)=\int_{0}^{1} b_{i}(s) u(s) \nabla s, 1 \leq i \leq n,
\end{gathered}
$$

has a unique solution

$$
u(t)=\int_{0}^{1} K_{i}(t, s) h(s) \nabla s, \text { for } 1 \leq i \leq n
$$

where,

$$
K_{i}(t, s)=G_{i}(t, s)+\theta_{i}(t) \int_{0}^{1} G_{i}(r, s) a_{j}(r) \nabla r+\zeta_{i}(t) \int_{0}^{1} G_{i}(r, s) b_{i}(r) \nabla r,
$$

for $1 \leq i \leq n$,

Proof. Suppose that $\mathrm{u}$ is a solution of (3.1), then, we have

$$
\begin{aligned}
u(t) & =-\int_{0}^{t} \int_{0}^{s} h(r) \nabla r \Delta s+A t+B \\
& =-\int_{0}^{t}(t-s) h(s) \nabla s+A t+B
\end{aligned}
$$

where $A=\lim _{t \rightarrow 0^{+}} u^{\Delta}(t)$ and $B=u(0)$. Using the boundary conditions (3.1), (3.2) we can determined $A$ and $B$ as

$$
\begin{aligned}
& A=\frac{1}{d_{i}} \int_{0}^{1}\left[\alpha_{i} b_{i}(s)-\gamma_{i} a_{i}(s)\right] u(s) \nabla s+\frac{\alpha_{i}}{d_{i}} \int_{0}^{1}\left[\gamma_{i}(1-s)+\delta_{i}\right] h(s) \nabla s \\
& B=\frac{1}{d_{i}} \int_{0}^{1}\left[\left(\gamma_{i}+\delta_{i}\right) a_{i}(s)+\beta_{i} b_{i}(s)\right] u(s) \nabla s+\frac{\beta_{i}}{d_{i}} \int_{0}^{1}\left[\gamma_{i}(1-s)+\delta_{i}\right] h(s) \nabla s
\end{aligned}
$$

Thus, we have

$$
\begin{aligned}
u(t)=\frac{1}{d_{i}}\left[\int_{0}^{t}\left(\gamma_{i}+\delta_{i}-\gamma_{i} t\right)\left(\beta_{i}+\alpha_{i} s\right) h(s) \nabla s+\int_{t}^{1}\left(\gamma_{i}+\delta_{i}-\gamma_{i} s\right)\left(\beta_{i}+\alpha_{i} t\right) h(s) \nabla s\right] \\
+\frac{1}{d_{i}}\left[\int_{0}^{1}\left[\left(\gamma_{i}+\delta_{i}-\gamma_{i} t\right) a_{i}(s)+\left(\beta_{i}+\alpha_{i} t\right) b_{i}(s)\right] u(s) \nabla s\right] \\
=\frac{1}{d_{i}}\left[\int_{0}^{t} \phi_{i}(t) \psi_{i}(s) h(s) \nabla s+\int_{t}^{1} \phi_{i}(s) \psi_{i}(t) h(s) \nabla s\right] \\
+\frac{1}{d_{i}} \int_{0}^{1}\left[\phi_{i}(t) a_{i}(s)+\psi_{i}(t) b_{i}(s)\right] u(s) \nabla s,
\end{aligned}
$$

from which, we obtain

$$
u(t)=\int_{0}^{1} G_{i}(t, s) h(s) \nabla s+\frac{1}{d_{i}} \phi_{i}(t) \int_{0}^{1} a_{i}(s) u(s) \nabla s+\frac{1}{d_{i}} \psi_{i}(t) \int_{0}^{1} b_{i}(s) u(s) \nabla s .
$$

After certain computations we can determined,

$$
\begin{aligned}
\int_{0}^{1} a_{i}(s) u(s) \nabla s & =\frac{\left(1-v_{i}\right) A_{i}+u_{i}^{*} B_{i}}{\left(1-u_{i}\right)\left(1-v_{i}\right)-v_{i}^{*} u_{i}^{*}} . \\
\int_{0}^{1} b_{i}(s) u(s) \nabla s & =\frac{v_{i}^{*} A_{i}+\left(1-u_{i}\right) B_{i}}{\left(1-u_{i}\right)\left(1-v_{i}\right)-v_{i}^{*} u_{i}^{*}} .
\end{aligned}
$$


From (3.7) and (3.8), (3.6) can be written as

$$
\begin{aligned}
u(t)= & \int_{0}^{1} G_{i}(t, s) h(s) \nabla s+\frac{1}{d_{i}} \phi_{i}(t) \frac{\left(1-v_{i}\right) A_{i}+u_{i}^{*} B_{i}}{\left(1-u_{i}\right)\left(1-v_{i}\right)-v_{i}^{*} u_{i}^{*}} \\
& +\frac{1}{d_{i}} \psi_{i}(t) \frac{v_{i}^{*} A_{i}+\left(1-u_{i}\right) B_{i}}{\left(1-u_{i}\right)\left(1-v_{i}\right)-v_{i}^{*} u_{i}^{*}} \\
= & \int_{0}^{1} G_{i}(t, s) h(s) \nabla s+\frac{\left(1-v_{i}\right) \phi_{i}(t)+v_{i}^{*} \psi_{i}(t)}{d_{i}\left(\left(1-u_{i}\right)\left(1-v_{i}\right)-v_{i}^{*} u_{i}^{*}\right)} A_{i} \\
& +\frac{\left(1-u_{i}\right) \psi_{i}(t)+u_{i}^{*} \phi_{i}(t)}{d_{i}\left(\left(1-u_{i}\right)\left(1-v_{i}\right)-v_{i}^{*} u_{i}^{*}\right)} B_{i} \\
= & \int_{0}^{1} G_{i}(t, s) h(s) \nabla s+\theta_{i}(t) A_{i}+\zeta_{i}(t) B_{i} \quad+\zeta_{i}(t) \int_{0}^{1}\left(\int_{0}^{1} G_{i}(r, s) b_{i}(r) \nabla r\right) h(s) \nabla s \\
= & \int_{0}^{1} G_{i}(t, s) h(s) \nabla s+\theta_{i}(t) \int_{0}^{1}\left(\int_{0}^{1} G_{i}(r, s) a_{i}(r) \nabla r\right) h(s) \nabla s \\
= & \int_{0}^{1}\left[G_{i}(t, s)+\theta_{i}(t) \int_{0}^{1} G_{i}(r, s) a_{i}(r) \nabla r\right. \\
& \left.+\zeta_{i}(t) \int_{0}^{1} G_{i}(r, s) b_{i}(r) \nabla r\right] h(s) \nabla s \\
= & \int_{0}^{1} K_{i}(t, s) h(s) \nabla s, \quad
\end{aligned}
$$

where $K_{i}(t, s)$ is defined in (3.5). The proof is complete.

Lemma 3.2. Assume that $(H 4),(H 5)$ hold and for $\tau \in\left(0, \frac{1}{2}\right) \mathbb{T}$ define $\eta_{i}(\tau)=\min \left\{\frac{\alpha_{i} \tau+\beta_{i}}{\alpha_{i}+\beta_{i}}, \frac{\gamma_{i} \tau+\delta_{i}}{\gamma_{i}+\delta_{i}}\right\}<1$. Then $G_{i}(t, s)$ for $1 \leq i \leq n$, satisfies the following properties:

(i) $0 \leq G_{i}(t, s) \leq G_{i}(s, s)$ for all $t, s \in J_{0}$,

(ii) $0 \leq \eta_{i}(\tau) G_{i}(s, s) \leq G_{i}(t, s)$ for all $t \in[\tau, 1-\tau] \mathbb{T}$ and $s \in J_{0}$,

Lemma 3.3. Assume that (H4), (H5) holds. Then $K_{i}(t, s)$ for $1 \leq i \leq n$, have the following properties:

(i) $0 \leq K_{i}(t, s) \leq\left(1+\theta_{i}^{*} a_{i}^{*}+\zeta_{i}^{*} b_{i}^{*}\right) G_{i}(s, s)$ for all $t, s \in J_{0}$,

(ii) $0 \leq \eta_{i}(\tau)\left(1+\theta_{i}^{* *} a_{i}(\tau)+\zeta_{i}^{* *} b_{i}(\tau)\right) G_{i}(s, s) \leq K_{i}(t, s)$ for all $t \in[\tau, 1-\tau] \mathbb{T}$ and $s \in J_{0}$

Lemma 3.4. Assume that $(H 4),(H 5)$ hold and $K_{j}(t, s)$ for $1 \leq j \leq n$, is given in (3.5). Let $H_{1}(t, s)=$ $K_{1}(t, s)$ and recursively define

$$
H_{j}(t, s)=\int_{0}^{1} H_{j-1}(t, r) K_{j}(r, s) \nabla r, \quad \text { for } \quad 2 \leq j \leq n .
$$

Then $H_{n}(t, s)$ is the Green's function for the homogeneous boundary value problem

$$
\begin{gathered}
(-1)^{n} u^{(\Delta \nabla)^{n}}(t)=0, t \in J_{0}, \\
\alpha_{i+1} u^{(\Delta \nabla)^{i}}(0)-\beta_{i+1} u^{(\Delta \nabla)^{i} \Delta}(0)=\int_{0}^{1} a_{i+1}(s) u^{(\Delta \nabla)^{i}}(s) \nabla s, 0 \leq i \leq n-1, \\
\gamma_{i+1} u^{(\Delta \nabla)^{i}}(1)+\delta_{i+1} u^{(\Delta \nabla)^{i} \Delta}(1)=\int_{0}^{1} b_{i+1}(s) u^{(\Delta \nabla)^{i}}(s) \nabla s, 0 \leq i \leq n-1 .
\end{gathered}
$$


Lemma 3.5. Assume that $(H 4),(H 5)$ hold. Define $g=\prod_{j=1}^{n-1} g_{j}$,

$$
\phi=\prod_{j=1}^{n}\left(1+\theta_{j}^{*} a_{j}^{*}+\zeta_{j}^{*} b_{j}^{*}\right), \eta_{\tau}=\prod_{j=1}^{n} \eta_{j}(\tau)\left(1+\theta_{j}^{* *} a_{j}(\tau)+\zeta_{j}^{* *} b_{j}(\tau)\right), g_{\tau}=\prod_{j=1}^{n-1} g_{j}(\tau)
$$

then the Green's function $H_{n}(t, s)$ satisfies the following inequalities:

(i) $0 \leq H_{n}(t, s) \leq g \phi G_{n}(s, s)$, for all $t, s \in J_{0}$ and

(ii) $H_{n}(t, s) \geq \eta_{\tau} g_{\tau} G_{n}(s, s)$, for all $t \in[\tau, 1-\tau]_{\mathbb{T}}$ and $s \in J_{0}$,

Proof. It is clear that Green's function $H_{n}(t, s) \geq 0$, for all $t, s \in J_{0}$. Now we prove the inequality by induction on $n$ and denote the statement by $p(n)$. From (3.5) we have

$$
\begin{aligned}
H_{1}(t, s) & =G_{1}(t, s)+\theta_{1}(t) \int_{0}^{1} G_{1}(r, s) a_{1}(r) \nabla r+\zeta_{1}(t) \int_{0}^{1} G_{1}(r, s) b_{1}(r) \nabla r \\
& \leq G_{1}(s, s)+\theta_{1}(t) \int_{0}^{1} G_{1}(s, s) a_{1}(r) \nabla r+\zeta_{1}(t) \int_{0}^{1} G_{1}(s, s) b_{1}(r) \nabla r \\
& \leq\left(1+\theta_{1}(t) \int_{0}^{1} a_{1}(r) \nabla r+\zeta_{1}(r) \int_{0}^{1} b_{1}(r) \nabla r\right) G_{1}(s, s) \\
& \leq\left(1+\theta_{1}^{*} a_{1}^{*}+\zeta_{1}^{*} b_{1}^{*}\right) G_{1}(s, s)
\end{aligned}
$$

and for $t \in[\tau, 1-\tau] \mathbb{T}$,

$$
\begin{aligned}
H_{1}(t, s) & =G_{1}(t, s)+\theta_{1}(t) \int_{0}^{1} G_{1}(r, s) a_{1}(r) \nabla r+\zeta_{1}(t) \int_{0}^{1} G_{1}(r, s) b_{1}(r) \nabla r \\
& \geq G_{1}(t, s)+\theta_{1}(t) \int_{\tau}^{1-\tau} G_{1}(r, s) a_{1}(r) \nabla r+\zeta_{1}(t) \int_{\tau}^{1-\tau} G_{1}(r, s) b_{1}(r) \nabla r \\
& \geq \eta_{1}(\tau) G_{1}(s, s)+\theta_{1}(t) \eta_{1}(\tau) G_{1}(s, s) \int_{\tau}^{1-\tau} a_{1}(r) \nabla r \\
& \quad+\zeta_{1}(t) \eta_{1}(\tau) G_{1}(s, s) \int_{\tau}^{1-\tau} b_{1}(r) \nabla r \\
\geq & \left(1+\theta_{1}(t) \int_{\tau}^{1-\tau} a_{1}(r) \nabla r+\zeta_{1}(r) \int_{\tau}^{1-\tau} b_{1}(r) \nabla r\right) \eta_{1}(\tau) G_{1}(s, s) \\
\geq & \left(1+\theta_{1}^{* *} a_{1}(\tau)+\zeta_{1}^{* *} b_{1}(\tau)\right) \eta_{1}(\tau) G_{1}(s, s)
\end{aligned}
$$

Hence, $p(1)$ is true. From (3.9), we have

$$
\begin{aligned}
H_{m+1}(t, s) & =\int_{0}^{1} H_{m}(t, r) K_{m+1}(r, s) \nabla r \\
& \leq \int_{0}^{1} G_{m}(r, r) g \phi\left(1+\theta_{m+1}^{*} a_{m+1}^{*}+\zeta_{m+1}^{*} b_{m+1}^{*}\right) G_{m+1}(s, s) \nabla r \\
& \leq\left(\int_{0}^{1} G_{m}(r, r) \nabla r\right) g \phi\left(1+\theta_{m+1}^{*} a_{m+1}^{*}+\zeta_{m+1}^{*} b_{1}^{*}\right) G_{m+1}(s, s) \\
& \leq\left(\prod_{i=1}^{m} g_{i}\right) \prod_{i=1}^{m+1}\left(1+\theta_{i}^{*} a_{i}^{*}+\zeta_{i}^{*} b_{i}^{*}\right) G_{m+1}(s, s)
\end{aligned}
$$


and for $t \in[\tau, 1-\tau]_{\mathbb{T}}$,

$$
\begin{aligned}
H_{m+1}(t, s) & =\int_{0}^{1} H_{m}(t, r) K_{m+1}(r, s) \nabla r \\
& \geq \int_{\tau}^{1-\tau} \eta_{\tau} g_{\tau} G_{m}(r, r) \eta_{m+1}(\tau)\left(1+\theta_{m+1}^{* *} a_{m+1}(\tau)+\zeta_{m+1}^{* *} b_{m+1}(\tau)\right) G_{m+1}(s, s) \nabla r \\
& \geq g_{\tau}\left(\int_{\tau}^{1-\tau} G_{m}(r, r) \nabla r\right) \eta_{\tau} \eta_{m+1}(\tau)\left(1+\theta_{m+1}^{* *} a_{m+1}(\tau)+\zeta_{m+1}^{* *} b_{m+1}(\tau)\right) G_{m+1}(s, s) \\
& \geq\left(\prod_{i=1}^{m} g_{i}(\tau)\right) \prod_{i=1}^{m+1} \eta_{m+1}(\tau)\left(1+\theta_{i}^{* *} a_{i}(\tau)+\zeta_{i}^{* *} b_{i}(\tau)\right) G_{m+1}(s, s)
\end{aligned}
$$

So, $p(m+1)$ holds. Thus $p(n)$ is true by induction

Let $X$ denotes the Banach space $C_{l d}\left(J_{0}, \mathbb{R}\right)$ with norm $\|u\|=\max _{t \in J_{0}}|u(t)|$. For $\tau \in\left(0, \frac{1}{2}\right) \mathbb{T}$, define the cone $P_{\tau} \subset X$ by

$$
P_{\tau}=\left\{u \in X: u(t) \geq 0 \text { and } \min _{t \in[\tau, 1-\tau]} u(t) \geq \xi_{\tau}\|u(t)\|\right\},
$$

where $\xi_{\tau}=\frac{\eta_{\tau} g_{\tau}}{g \phi}$. For any $u \in P_{\tau}$, define an operator $T: P_{\tau} \rightarrow X$ by

$$
(T u)(t)=\int_{0}^{1} H_{n}(t, s) \omega(s) f(u(s)) \nabla s .
$$

Lemma 3.6. Assume that (H1)-(H3) hold. Then $T\left(P_{\tau}\right) \subset P_{\tau}$ and $T: P_{\tau} \rightarrow P_{\tau}$ is completely continuous for each $\tau \in\left(0, \frac{1}{2}\right) \mathbb{T}$.

Proof. Fix $\tau \in\left(0, \frac{1}{2}\right)$. Since $\omega(s) f(u(s)) \geq 0$ for all $s \in J_{0}, u \in P_{\tau}$ and since $H_{n}(t, s) \geq 0$ for all $t, s \in J_{0}$, then $T(u(t)) \geq 0$ for all $t \in J_{0}, u \in P_{\tau}$. On the other hand, by Lemma 3.5 we obtain

$$
\begin{aligned}
(T u)(t) & =\int_{0}^{1} H_{n}(t, s) \omega(s) f(u(s)) \nabla s \\
& \leq g \phi \int_{0}^{1} G_{n}(s, s) \omega(s) f(u(s)) \nabla s \\
\min _{t \in[\tau, 1-\tau]} u(t) & =\min _{t \in[\tau, 1-\tau]} \int_{0}^{1} H_{n}(t, s) \omega(s) f(u(s)) \nabla s \\
& \geq \int_{0}^{1} \min _{t \in[\tau, 1-\tau]} H_{n}(t, s) \omega(s) f(u(s)) \nabla s \\
& =\eta_{\tau} g_{\tau} \int_{0}^{1} G_{n}(s, s) \omega(s) f(u(s)) \nabla s \\
& \geq \xi_{\tau} T u(t)
\end{aligned}
$$

for all $t \in J_{0}$. Thus $\min _{t \in[\tau, 1-\tau]} u(t) \geq \xi_{\tau}\|T u\|$. So, $T u \in P_{\tau}$ and then $T\left(P_{\tau}\right) \subset P_{\tau}$. Next, by standard methods and the Arzela-Ascoli theorem, one can easily prove that the operator $T$ is completely continuous. The proof is complete.

\section{MAin Results}

In this section, we establish the existence of countably infinitely many positive solutions for the boundary value problem (1.6)-(1.7) by applying Krasnoselskii's fixed point theorem in cones.

Theorem 4.1. [14] Let $\mathcal{B}$ be a Banach space and let $P \subset \mathcal{B}$ be a cone in $\mathcal{B}$. Assume that $\Omega_{1}, \Omega_{2}$ are open with $0 \in \Omega_{1}, \bar{\Omega}_{1} \subset \Omega_{2}$, and let $T: P \cap\left(\bar{\Omega}_{2} \backslash \Omega_{1}\right) \rightarrow P$ be a completely continuous operator such that either

(i) $\|T u\| \leq\|u\|, u \in P \cap \partial \Omega_{1}$, and $\|T u\| \geq\|u\|, u \in P \cap \partial \Omega_{2}$, or 
(ii) $\|T u\| \geq\|u\|, u \in P \cap \partial \Omega_{1}$, and $\|T u\| \leq\|u\|, u \in P \cap \partial \Omega_{2}$.

Then $T$ has a fixed point in $P \cap\left(\bar{\Omega}_{2} \backslash \Omega_{1}\right)$.

Theorem 4.2. [4, 29] Let $f \in L_{\nabla}^{p}(J)$ with $p>1, g \in L_{\nabla}^{q}(J)$ with $q>1$, and $\frac{1}{p}+\frac{1}{q}=1$. Then $f g \in L_{\nabla}^{1}(J)$ and $\|f g\|_{L_{\nabla}^{1}} \leq\|f\|_{L_{\nabla}^{p}}\|g\|_{L_{\nabla}^{q}}$.

where

$$
\|f\|_{L_{\nabla}^{p}}:= \begin{cases}{\left[\int_{J}|f|^{p}(s) \nabla s\right]^{\frac{1}{p}},} & p \in \mathbb{R}, \\ \inf \{M \in \mathbb{R} /|f| \leq M \nabla-\text { a.e., on } J\}, & p=\infty,\end{cases}
$$

and $J=(a, b]$. Moreover, if $f \in L_{\nabla}^{1}(J)$ and $g \in L_{\nabla}^{\infty}(J)$. Then $f g \in L_{\nabla}^{1}(J)$ and $\|f g\|_{L_{\nabla}^{1}} \leq\|f\|_{L_{\nabla}^{1}}\|g\|_{L_{\nabla}^{\infty}}$.

We consider the following three cases for $\omega \in L_{\nabla}^{p}\left(J_{0}\right): p>1, p=1, p=\infty$. Case $p>1$ is treated in the following theorem.

Theorem 4.3. Assume that (H1)-(H5) hold, let $\left\{\tau_{k}\right\}_{k=1}^{\infty}$ be such that $t_{k+1}<\tau_{k}<t_{k}, k=1,2,3, \cdots$. Let $\left\{R_{k}\right\}_{k=1}^{\infty}$ and $\left\{r_{k}\right\}_{k=1}^{\infty}$ be such that

$$
R_{k+1}<\xi_{\tau_{k}} r_{k}<C r_{k}<R_{k}, k \in \mathbb{N},
$$

where

$$
C=\max \left\{\frac{1}{\eta_{\tau_{1}} g_{\tau_{1}} m \int_{\tau_{1}}^{1-\tau_{1}} G_{n}(s, s) \nabla s}, 1\right\}
$$

Assume that $f$ satisfies

(A1) $f(u) \leq M_{1} R_{k}$ for all $t \in J_{0}, 0 \leq u \leq R_{k}$, where $M_{1}<\frac{1}{g \phi\left\|G_{n}(s, s)\right\|_{L_{\nabla}^{q}}\|\omega\|_{L_{\nabla}^{p}}}$,

(A2) $f(u) \geq C r_{k}$ for all $t \in\left[\tau_{k}, 1-\tau_{k}\right]_{\mathbb{T}}, \xi_{\tau} r_{k} \leq u \leq r_{k}$.

Then the boundary value problem (1.6)-(1.7) has countably infinitely many positive solutions $\left\{u_{k}\right\}_{k=1}^{\infty}$. Furthermore, $r_{k} \leq\left\|u_{k}\right\| \leq R_{k}$ for each $k \in \mathbb{N}$.

Proof. Consider the sequences $\left\{\Omega_{1, k}\right\}_{k=1}^{\infty}$ and $\left\{\Omega_{2, k}\right\}_{k=1}^{\infty}$ of open subsets of $E$ defined by

$$
\begin{gathered}
\Omega_{1, k}=\left\{u \in \mathcal{B}:\|u\|<R_{k}\right\}, \\
\Omega_{2, k}=\left\{u \in \mathcal{B}:\|u\|<r_{k}\right\} .
\end{gathered}
$$

Let $\left\{\tau_{k}\right\}_{k=1}^{\infty}$ be as in the hypothesis and note that $t^{*}<t_{k+1}<\tau_{k}<t_{k}<\frac{1}{2}$, for all $k \in \mathbb{N}$. For each $k \in \mathbb{N}$, define the cone $P_{\tau_{k}}$ by

$$
P_{\tau_{k}}=\left\{u \in X: u(t) \geq 0 \text { and } \min _{t \in\left[\tau_{k}, 1-\tau_{k}\right]} u(t) \geq \xi_{\tau_{k}}\|u(t)\|\right\} .
$$

Let $u \in P_{\tau_{k}} \cap \partial \Omega_{1, k}$. Then,

$$
u(s) \leq R_{k}=\|u\|
$$

for all $s \in J_{0}$. By $(A 1)$,

$$
\begin{aligned}
\|T u\| & =\max _{t \in J_{0}} \int_{0}^{1} H_{n}(t, s) \omega(s) f(u(s)) \nabla s \\
& \leq g \phi \int_{0}^{1} G_{n}(s, s) \omega(s) \nabla s M_{1} R_{k} \\
& \leq g \phi\left\|G_{n}(s, s)\right\|_{L_{\nabla}^{q}}\|\omega\|_{L_{\nabla}^{p}} M_{1} R_{k} \\
& \leq R_{k} .
\end{aligned}
$$

Since $\|u\|=R_{k}$ for all $u \in P_{\tau_{k}} \cap \partial \Omega_{1, k}$, then

$$
\|T u\| \leq\|u\| .
$$

Let $s \in\left[\tau_{k}, 1-\tau_{k}\right] \mathbb{T}$. Then,

$$
r_{k}=\|u\| \geq u(s) \geq \min _{s \in\left[\tau_{k}, 1-\tau_{k}\right]_{\mathbb{T}}} u(s) \geq \xi_{\tau}\|u\| \geq \xi_{\tau_{k}} r_{k} .
$$


By $(A 2)$,

$$
\begin{aligned}
\|T u\| & =\max _{t \in J_{0}} \int_{0}^{1} H_{n}(t, s) \omega(s) f(u(s)) \nabla s \\
& \geq \max _{t \in J_{0}} \int_{\tau_{k}}^{1-\tau_{k}} H_{n}(t, s) \omega(s) f(u(s)) \nabla s \\
& \geq \max _{t \in J_{0}} \int_{\tau_{k}}^{1-\tau_{k}} H_{n}(t, s) \omega(s) \nabla s C r_{k} \\
& \geq C r_{k} m \max _{t \in\left[\tau_{1}, 1-\tau_{1}\right]} \int_{\tau_{1}}^{1-\tau_{1}} H_{n}(t, s) \nabla s \\
& \geq \eta_{\tau_{1}} g_{\tau_{1}} m C r_{k} \max _{t \in\left[\tau_{1}, 1-\tau_{1}\right]} \int_{\tau_{1}}^{1-\tau_{1}} G_{n}(s, s) \nabla s \\
& \geq r_{k}=\|u\| .
\end{aligned}
$$

Thus, if $u \in P_{\tau} \cap \partial \Omega_{2, k}$, then

$$
\|T u\| \geq\|u\|
$$

It is obvious that $0 \in \Omega_{2, k} \subset \bar{\Omega}_{2, k} \subset \Omega_{1, k}$. By (4.1),(4.2), it follows from Theorem 4.1 that the operator $T$ has a fixed point $u_{k} \in P_{\tau_{k}} \cap\left(\bar{\Omega}_{1, k} \backslash \Omega_{2, k}\right)$ such that $r_{k} \leq\left\|u_{k}\right\| \leq R_{k}$. Since $k \in \mathbb{N}$ was arbitrary, the proof is complete.

Now we deal with the case $p=1$.

Theorem 4.4. Assume that (H1)-(H5) hold, let $\left\{\tau_{k}\right\}_{k=1}^{\infty}$ be such that $t_{k+1}<\tau_{k}<t_{k}, k=1,2,3, \cdots$. Let $\left\{R_{k}\right\}_{k=1}^{\infty}$ and $\left\{r_{k}\right\}_{k=1}^{\infty}$ be such that

$$
R_{k+1}<\xi_{\tau_{k}} r_{k}<C r_{k}<R_{k}, k \in \mathbb{N},
$$

Assume that $f$ satisfies

(B1) $f(u) \leq M_{2} R_{k}$ for all $t \in J_{0}, 0 \leq u \leq R_{k}$, where

$$
M_{2}<\min \left\{\frac{1}{g \phi\left\|G_{n}(s, s)\right\|_{L_{\nabla}^{\infty}}\|\omega\|_{L_{\nabla}^{1}}}, C\right\}
$$

and (A2). Then the boundary value problem (1.6)-(1.7) has countably infinitely many positive solutions $\left\{u_{k}\right\}_{k=1}^{\infty}$. Furthermore, $r_{k} \leq\left\|u_{k}\right\| \leq R_{k}$ for each $k \in \mathbb{N}$.

Proof. For a fixed $k$, let $\Omega_{1, k}$ be as in the proof of Theorem 4.3 and let $u \in P_{\tau_{k}} \cap \partial \Omega_{2, k}$. Again

$$
u(s) \leq A_{k}=\|u\|,
$$

for all $s \in J_{0}$. By $(B 1)$ and Theorem 4.3,

$$
\begin{aligned}
\|T u\| & =\max _{t \in J_{0}} \int_{0}^{1} H_{n}(t, s) \omega(s) f(u(s)) \nabla s \\
& \leq g \phi \int_{0}^{1} G_{n}(s, s) \omega(s) \nabla s M_{2} R_{k} \\
& \leq g \phi\left\|G_{n}(s, s)\right\|_{L_{\nabla}^{\infty}}\|\omega\|_{L_{\nabla}^{1}} M_{2} R_{k} \\
& \leq R_{k} .
\end{aligned}
$$

Thus,

$$
\|T u\| \leq\|u\|
$$

for $u \in P_{\tau_{k}} \cap \partial \Omega_{1, k}$. Now define $\Omega_{2, k}=\left\{u \in \mathcal{B}:\|u\|<r_{k}\right\}$. Let $u \in P_{\tau_{k}} \cap \partial \Omega_{2, k}$ and let $s \in\left[\tau_{k}, 1-\tau_{k}\right] \mathbb{T}$. Then, the argument leading to (4.2) carries over to the present case and completes the proof.

Finally we consider the case of $p=\infty$. 
Theorem 4.5. Assume that $(H 1)-(H 5)$ hold. Let $\left\{R_{k}\right\}_{k=1}^{\infty}$ and $\left\{r_{k}\right\}_{k=1}^{\infty}$ be such that

$$
R_{k+1}<\xi_{\tau} r_{k}<C r_{k}<R_{k}, k \in \mathbb{N},
$$

Assume that $f$ satisfies

(E1) $f(u) \leq M_{3} R_{k}$ for all $t \in J_{0}, 0 \leq u \leq R_{k}$, where

$$
M_{3}<\min \left\{\frac{1}{g \phi\left\|G_{n}(s, s)\right\|_{L_{\nabla}^{1}}\|\omega\|_{L_{\nabla}^{\infty}}}, C\right\}
$$

and (A2). Then the boundary value problem (1.6)-(1.7) has countably infinitely many positive solutions $\left\{u_{k}\right\}_{k=1}^{\infty}$. Furthermore, $r_{k} \leq\left\|u_{k}\right\| \leq R_{k}$ for each $k \in \mathbb{N}$.

Proof. By (E1),

$$
\begin{aligned}
\|T u\| & =\max _{t \in J_{0}} \int_{0}^{1} H_{n}(t, s) \omega(s) f(u(s)) \nabla s \\
& \leq g \phi \int_{0}^{1} G_{n}(s, s) \omega(s) \nabla s M_{3} R_{k} \\
& \leq g \phi\left\|G_{n}(s, s)\right\|_{L_{\nabla}^{1}}\|\omega\|_{L_{\nabla}^{\infty}} M_{3} R_{k} \\
& \leq R_{k} .
\end{aligned}
$$

This shows that if $u \in P_{\tau_{k}} \cap \partial \Omega_{1, k}$, where $\Omega_{1, k}=\left\{u \in \mathcal{B}:\|u\|<R_{k}\right\}$, Then,

$$
\|T u\| \leq\|u\| \text {. }
$$

Define $\Omega_{2, k}=\left\{u \in \mathcal{B}:\|u\|<r_{k}\right\}$ and let $u \in P_{\tau_{k}} \cap \partial \Omega_{2, k}$. Then, the argument employed in the proof of Theorem 4.3 applies directly to yield

$$
\|T u\| \geq\|u\|
$$

By the Theorem 4.1, completes the proof.

\section{Example}

In this section, we provide an example of a family of functions $\omega(t)$ that satisfy conditions $(H 1),(H 2)$ corresponding to the cases $p=1$ and $p=2$. Let $\mathbb{T}=\left[0, \frac{1}{2}\right] \cup\left\{\frac{3}{5}, \frac{3}{4}, \frac{4}{5}\right\} \cup\left[\frac{5}{6}, 1\right]$ and consider the family of functions $\omega(t, \epsilon): \mathbb{T} \rightarrow(0,+\infty]$ given by

$$
\omega(t, \epsilon)=\left\{\begin{array}{cc}
\sum_{k=1}^{\infty} \frac{\chi\left[\nu_{k}, \nu_{k-1}\right]}{\left|t-t_{k}\right|^{\epsilon}} & \text { if } 0 \leq t \leq \frac{1}{2}, \\
\frac{1}{\left|t-\frac{1}{2}\right|^{\epsilon}} & \text { if } \frac{1}{2}<t<\frac{5}{6} \\
\frac{1}{\left|t-\frac{4}{5}\right|^{\epsilon}} & \text { if } \frac{5}{6} \leq t \leq 1,
\end{array}\right.
$$

where

$$
\begin{gathered}
t_{0}=\frac{5}{16}, t_{k}=t_{0}-\sum_{i=0}^{k-1} \frac{1}{(i+2)^{4}}, k=1,2,3, \cdots, \\
\nu_{0}=1, \nu_{k}=\frac{1}{2}\left(t_{k}+t_{k+1}\right), k=1,2,3, \cdots .
\end{gathered}
$$

At first, it is easily seen that $\omega(t, \epsilon) \geq \omega(1, \epsilon)=\frac{1}{\left|1-\frac{4}{5}\right|^{\epsilon}}=5^{\epsilon}, t_{1}=\frac{1}{4}<\frac{1}{2}, t_{k}-t_{k+1}=\frac{1}{(k+2)^{4}}, k=$ $1,2,3, \cdots$, and note that $\sum_{k=1}^{\infty} \frac{1}{k^{4}}=\frac{\pi^{4}}{90}$.

$$
t^{*}=\lim _{k \rightarrow \infty} t_{k}=\frac{5}{16}-\sum_{i=0}^{\infty} \frac{1}{(i+2)^{4}}=\frac{5}{16}-\left(\frac{\pi^{4}}{90}-1\right)=\frac{21}{16}-\frac{\pi^{4}}{90}>\frac{1}{5} .
$$


We claim that if $\epsilon=\frac{1}{2}$, then $\omega(t, \epsilon) \in L_{\nabla}^{1}[0,1]$. Note that $\sum_{k=1}^{\infty} \frac{1}{k^{2}}=\frac{\pi^{2}}{6}$, we have

$$
\begin{aligned}
& \int_{0}^{1} \omega(t, \epsilon) \nabla t=\int_{0}^{\frac{1}{2}} \omega(t, \epsilon) \nabla t+\int_{\frac{1}{2}}^{1} \omega(t, \epsilon) \nabla t \\
& =\int_{0}^{\frac{1}{2}} \sum_{k=1}^{\infty} \frac{\chi\left[\nu_{k}, \nu_{k-1}\right]}{\left|t-t_{k}\right|^{\epsilon}} \nabla t+\int_{\frac{5}{6}}^{1} \frac{1}{\left|t-\frac{4}{5}\right| \epsilon} \nabla t+\left[\left(\frac{3}{5}-\frac{1}{2}\right) \omega\left(\frac{3}{5}, \epsilon\right)\right. \\
& \left.+\left(\frac{3}{4}-\frac{3}{5}\right) \omega\left(\frac{3}{4}, \epsilon\right)+\left(\frac{4}{5}-\frac{3}{4}\right) \omega\left(\frac{4}{5}, \epsilon\right)\left(\frac{5}{6}-\frac{4}{5}\right) \omega\left(\frac{5}{6}, \epsilon\right)\right] \\
& =\sum_{k=1}^{\infty} \int_{\nu_{k}}^{\nu_{k-1}} \frac{1}{\left|t-t_{k}\right|^{\epsilon}} \nabla t+\int_{\frac{5}{6}}^{1} \frac{1}{\left(t-\frac{4}{5}\right)^{\epsilon}} \nabla t \\
& +\left[\frac{1}{10} \times 10^{\epsilon}+\frac{3}{20} \times 4^{\epsilon}+\frac{1}{20} \times\left(\frac{10}{3}\right)^{\epsilon}+\frac{1}{30} \times 3^{\epsilon}\right] \\
& =\sum_{k=1}^{\infty}\left[\int_{\nu_{k}}^{t_{k}} \frac{1}{\left(t_{k}-t\right)^{\epsilon}} \nabla t+\int_{t_{k}}^{\nu_{k-1}} \frac{1}{\left(t-t_{k}\right)^{\epsilon}} \nabla t\right]+\frac{1}{1-\epsilon}\left[\frac{1}{5^{1-\epsilon}}-\frac{1}{30^{1-\epsilon}}\right] \\
& +\left[\frac{1}{10} \times 10^{\epsilon}+\frac{3}{20} \times 4^{\epsilon}+\frac{1}{20} \times\left(\frac{10}{3}\right)^{\epsilon}+\frac{1}{30} \times 3^{\epsilon}\right] \\
& =\sum_{k=1}^{\infty}\left[\int_{\frac{t_{k}+t_{k+1}}{2}}^{t_{k}} \frac{1}{\left(t_{k}-t\right)^{\epsilon}} \nabla t+\int_{t_{k}}^{\frac{t_{k-1}+t_{k}}{2}} \frac{1}{\left(t-t_{k}\right)^{\epsilon}} \nabla t\right] \\
& +\frac{1}{1-\epsilon}\left[\frac{1}{5^{1-\epsilon}}-\frac{1}{30^{1-\epsilon}}\right]+\left[10^{\epsilon-1}+\frac{3}{5} \times 4^{\epsilon-1}+\frac{1}{20} \times\left(\frac{10}{3}\right)^{\epsilon}+\frac{1}{10} \times 3^{\epsilon-1}\right] \\
& =\frac{1}{1-\epsilon} \sum_{k=1}^{\infty}\left[\left(\frac{t_{k}-t_{k+1}}{2}\right)^{1-\epsilon}+\left(\frac{t_{k-1}-t_{k}}{2}\right)^{1-\epsilon}+\frac{1}{1-\epsilon}\left[\frac{1}{5^{1-\epsilon}}-\frac{1}{30^{1-\epsilon}}\right]\right. \\
& +\left[10^{\epsilon-1}+\frac{3}{5} \times 4^{\epsilon-1}+\frac{1}{20} \times\left(\frac{10}{3}\right)^{\epsilon}+\frac{1}{10} \times 3^{\epsilon-1}\right] \\
& =\frac{1}{2^{1-\epsilon}(1-\epsilon)} \sum_{k=1}^{\infty}\left[\frac{1}{(k+2)^{4(1-\epsilon)}}+\frac{1}{(k+1)^{4(1-\epsilon)}}\right]+\frac{1}{1-\epsilon}\left[\frac{1}{5^{1-\epsilon}}-\frac{1}{30^{1-\epsilon}}\right] \\
& +\left[10^{\epsilon-1}+\frac{3}{5} \times 4^{\epsilon-1}+\frac{1}{20} \times\left(\frac{10}{3}\right)^{\epsilon}+\frac{1}{10} \times 3^{\epsilon-1}\right] \\
& =\sqrt{2} \sum_{k=1}^{\infty}\left[\frac{1}{(k+1)^{2}}+\frac{1}{(k+1)^{2}}\right]+\frac{1}{15}(6 \sqrt{5}-\sqrt{30})+\frac{1}{60}[6(\sqrt{10}+3)+\sqrt{3}(\sqrt{10}+2)] \\
& =\sqrt{2}\left(\frac{\pi^{2}}{3}-\frac{9}{4}\right)+\frac{1}{60}[24 \sqrt{5}-3 \sqrt{30}+6 \sqrt{10}+2 \sqrt{3}+18],
\end{aligned}
$$

which implies that $\omega(t, \epsilon) \in L_{\nabla}^{1}[0,1]$.

Next, we claim that if $\epsilon=\frac{1}{4}$, then $\omega(t, \epsilon) \in L_{\nabla}^{2}[0,1]$. In this case, we need the cauchy product,

$$
\sum_{k=1}^{\infty} a_{k} \cdot \sum_{k=1}^{\infty} b_{k}=\sum_{k=1}^{\infty} c_{k},
$$

where

$$
c_{k}=\sum_{n=1}^{k} a_{n} b_{k-n+1} .
$$

Note that

$$
\int_{0}^{1} \omega^{2}(t, \epsilon) \nabla t=\int_{0}^{\frac{1}{2}}\left[\sum_{k=1}^{\infty} \frac{\chi\left[\nu_{k}, \nu_{k-1}\right]}{\left|t-t_{k}\right|^{\epsilon}}\right]^{2} \nabla t+\int_{\frac{1}{2}}^{1} \omega^{2}(t, \epsilon) \nabla t
$$


we use (5.1) and (5.2) and the fact that, if $X \cap Y=\emptyset$, then $\chi[X] \cdot \chi[Y]=0$ to simplify the integrand,

$$
\left[\sum_{k=1}^{\infty} \frac{\chi\left[\nu_{k}, \nu_{k-1}\right]}{\left|t-t_{k}\right|^{\epsilon}}\right]^{2}=\sum_{k=1}^{\infty} \sum_{n=1}^{k} \frac{\chi\left[\nu_{n}, \nu_{k-1}\right]}{\left|t-t_{n}\right|^{\epsilon}} \frac{\chi\left[\nu_{k-n+1}, \nu_{k-n}\right]}{\left|t-t_{k-n+1}\right|^{\epsilon}}=\sum_{k=1}^{\infty} \frac{\chi\left[\nu_{k}, \nu_{k-1}\right]}{\left|t-t_{k}\right|^{2 \epsilon}} \text { a.e., }
$$

and so (5.3) may be written as

$$
\begin{aligned}
& \int_{0}^{1} \omega^{2}(t, \epsilon) \nabla t=\sum_{k=1}^{\infty} \int_{0}^{\frac{1}{2}} \frac{\chi\left[\nu_{k}, \nu_{k-1}\right]}{\left|t-t_{k}\right|^{2 \epsilon}} \nabla t+\int_{\frac{1}{2}}^{1} \omega^{2}(t, \epsilon) \nabla t \\
& =\sum_{k=1}^{\infty} \int_{\nu_{k}}^{\nu_{k-1}} \frac{1}{\left|t-t_{k}\right|^{2 \epsilon}} \nabla t+\int_{\frac{5}{6}}^{1} \omega^{2}(t, \epsilon) \nabla t++\left[\left(\frac{3}{5}-\frac{1}{2}\right) \omega^{2}\left(\frac{3}{5}, \epsilon\right)\right. \\
& \left.+\left(\frac{3}{4}-\frac{3}{5}\right) \omega^{2}\left(\frac{3}{4}, \epsilon\right)+\left(\frac{4}{5}-\frac{3}{4}\right) \omega^{2}\left(\frac{4}{5}, \epsilon\right)\left(\frac{5}{6}-\frac{4}{5}\right) \omega^{2}\left(\frac{5}{6}, \epsilon\right)\right] \\
& =\sum_{k=1}^{\infty}\left[\int_{\nu_{k}}^{t_{k}} \frac{1}{\left(t_{k}-t\right)^{2 \epsilon}} \nabla t+\int_{t_{k}}^{\nu_{k-1}} \frac{1}{\left(t-t_{k}\right)^{2 \epsilon}} \nabla t\right]+\int_{\frac{5}{6}}^{1} \frac{1}{\left(t-\frac{4}{5}\right)^{2 \epsilon}} \nabla t \\
& +\left[\frac{1}{10} \times 10^{2 \epsilon}+\frac{3}{20} \times 4^{2 \epsilon}+\frac{1}{20} \times\left(\frac{10}{3}\right)^{2 \epsilon}+\frac{1}{30} \times 3^{2 \epsilon}\right] \\
& =\sum_{k=1}^{\infty}\left[\int_{\frac{t_{k}+t_{k+1}}{2}}^{t_{k}} \frac{1}{\left(t_{k}-t\right)^{2 \epsilon}} \nabla t+\int_{t_{k}}^{\frac{t_{k-1}+t_{k}}{2}} \frac{1}{\left(t-t_{k}\right)^{2 \epsilon}} \nabla t\right] \\
& +\frac{1}{1-2 \epsilon}\left[\frac{1}{5^{1-2 \epsilon}}-\frac{1}{30^{1-2 \epsilon}}\right]+\left[10^{2 \epsilon-1}+\frac{3}{5} \times 4^{2 \epsilon-1}+\frac{1}{20} \times\left(\frac{10}{3}\right)^{2 \epsilon}+\frac{1}{10} \times 3^{2 \epsilon-1}\right] \\
& =\frac{1}{1-2 \epsilon} \sum_{k=1}^{\infty}\left[\left(\frac{t_{k}-t_{k+1}}{2}\right)^{1-2 \epsilon}+\left(\frac{t_{k-1}-t_{k}}{2}\right)^{1-2 \epsilon}+\frac{1}{1-2 \epsilon}\left[\frac{1}{5^{1-2 \epsilon}}-\frac{1}{30^{1-2 \epsilon}}\right]\right. \\
& +\left[10^{2 \epsilon-1}+\frac{3}{5} \times 4^{2 \epsilon-1}+\frac{1}{20} \times\left(\frac{10}{3}\right)^{2 \epsilon}+\frac{1}{10} \times 3^{2 \epsilon-1}\right] \\
& =\frac{1}{2^{1-2 \epsilon}(1-2 \epsilon)} \sum_{k=1}^{\infty}\left[\frac{1}{(k+2)^{4(1-2 \epsilon)}}+\frac{1}{(k+1)^{4(1-2 \epsilon)}}\right]+\frac{1}{1-2 \epsilon}\left[\frac{1}{5^{1-2 \epsilon}}-\frac{1}{30^{1-2 \epsilon}}\right] \\
& +\left[10^{2 \epsilon-1}+\frac{3}{5} \times 4^{2 \epsilon-1}+\frac{1}{20} \times\left(\frac{10}{3}\right)^{2 \epsilon}+\frac{1}{10} \times 3^{2 \epsilon-1}\right] \\
& =\sqrt{2} \sum_{k=1}^{\infty}\left[\frac{1}{(k+1)^{2}}+\frac{1}{(k+1)^{2}}\right]+\frac{1}{15}(6 \sqrt{5}-5 \sqrt{6})+\frac{1}{60}[6(\sqrt{10}+3)+\sqrt{3}(\sqrt{10}+2)] \\
& =\sqrt{2}\left(\frac{\pi^{2}}{3}-\frac{9}{4}\right)+\frac{1}{60}[24 \sqrt{5}-20 \sqrt{6}+6(\sqrt{10}+3)+\sqrt{3}(\sqrt{10}+2)],
\end{aligned}
$$

which implies $\omega(t, \epsilon) \in L_{\nabla}^{2}[0,1]$.

\section{REFERENCES}

[1] R. P. Agarwal, M. Bohner, Basic calculus on time scales and some of its applications. Result Math, 35(1999), 3-22.

[2] R. P. Agarwal, V. Otero-Espinar, K. Perera and D.R. Vivero, Basic properties of Sobolev's spaces on time scales. Advan. Diff. Eqns., 2006(2006), Art. ID 038121.

[3] D. R. Anderson, I. Y. Karaca, Higher-order three-point boundary value problem on time scales. Comput. Math. Appl.56(2008), 2429-2443.

[4] G. A. Anastassiou, Intelligent mathematics: computational analysis. Vol. 5. Heidelberg: Springer, 2011.

[5] M. Bohner and H. Luo, Singular second-order multipoint dynamic boundary value problems with mixed derivatives, Adv. Diff. Eqns., 2006(2006), Art. ID 054989.

[6] M. Bohner, and A. Peterson, Dynamic equations on time scales: An introduction with applications. Birkhauser, Boston, (2001).

[7] M. Bohner, and A. Peterson, Advances in Dynamic Equations on Time Scales. Birkhauser, Boston, (2003).

[8] E. Cetin, F. S. Topal, Existence results for solutions of integral boundary value problems on time scales, Abstr. Appl. Anal., 2013(2013), Art. ID 708734. 
[9] F. T. Fen, and I. Y. Karaca, Existence of positive solutions for nonlinear second-order impulsive boundary value problems on time scales, Med. J. Math., 13(2016), 191-204.

[10] M. Feng, Existence of symmetric positive solutions for boundary value problem with integral boundary conditions, Appl. Math. Lett., 24(2011), 1419-1427.

[11] J. M. Gallardo, Second-order differential operators with integral boundary conditions and generation of analytic semigroups, Rocky Mountain J. Math., 30(2000), No. 4, 1265-1292.

[12] C. S. Goodrich, Existence of a positive solution to a nonlocal semipositone boundary value problem on a time scale, Comment. Math. Univ. Carol., 54(2013), No. 4, 509-525.

[13] C. S. Goodrich, On a first-order semipositone boundary value problem on a time scale, Appl. Anal. Disc. Math., 8 (2014), 269-287.

[14] D. Guo and V. Lakshmikantham, Nonlinear problems in abstract cones, Academic press, New York, 1988.

[15] Y. Guo, Y. Liu and Y. Liang, Positive solutions for the third order boundary value problems with the second derivatives, Bound. value probl., 2012(2012), Art. ID 34

[16] G. S. Guseinov, Integration on time scales. J. Math. Anal. App., 285(2003), No. 1, 107-127.

[17] N. A. Hamal and F. Yoruk, Symmetric positive solutions of fourth order integral BVP for an increasing homeomorphism and homomorphism with sign-changing nonlinearity on time scales, Comput. Math. Appl., 59(2010), No. 11, 3603-3611.

[18] M. Hu and L. Wang, Triple positive solutions for an impulsive dynamic equation with integral boundary condition on time scales, Inter. J. App. Math. Stat., 31(2013), No. 1, 67-78.

[19] I. Y. Karaca, Positive solutions for boundary value problems of second-order functional dynamic equations on time scales, Adv. Difference Equ. 21(2009) Art. ID 829735.

[20] I. Y. Karaca, F. Tokmak, Existence of positive solutions for third-order boundary value problems with integral boundary conditions on time scales, J. Ineq. App., 2013(2013), No. 1, 1-12.

[21] G. L. Karakostas and P. Ch. Tsamatos, Multiple positive solutions of some fredholm integral equations arisen from nonlocal boundary-value problems, Electron. J. Differential Equations, 30(2002), No. 30, 1-17.

[22] R. A. Khan, the generalized method of quasi-linearization and nonlinear boundary value problems with integral boundary conditions, Electron. J. Qual. Theory Differential Equations, 19(2003), No. 15, 1-15.

[23] V. Lakshmikantham, S. Sivasundaram, B. Kaymakcalan, Dynamic Systems on Measure Chains, Kluwer, Dordrecht, (1996).

[24] Y. Li and L. Sun, Infinite many positive solutions for nonlinear first-order BVPs with integral boundary conditions on time scales, Top. Meth. Nonl. Anal., 41(2013), No. 2, 305-321.

[25] Y. Li and L. Wang, Multiple positive solutions of nonlinear third-order boundary value problems with integral boundary conditions on time scales, Adv. Diff. Eqns., 2015(2015), Art. ID 90.

[26] Y. Li and T. Zhang, Multiple Positive Solutions for Second-Order p-Laplacian Dynamic Equations with Integral Boundary Conditions, Boun. Val. Prob., 2011(2010), Art. ID 19.

[27] A. Lomtatidze and L. Malaguti, On a nonlocal boundary-value problems for second order nonlinear singular differential equations, Georgian Math. J., 7(2000), 133-154.

[28] A. D. Oguz and F. S. Topal, Symmetric positive solutions for second order boundary value problems with integral boundary conditions on time scales, J. Appl. Anal and Comp., 6(2016), No. 2, 531-542.

[29] U. M. Ozkan and M. Z. Sarikaya and H. Yildirim, Extensions of certain integral inequalities on time scales. Appl. Math. Let., 21(2008), No. 10, 993-1000.

[30] B. P. Rynne, L2 spaces and boundary value problems on time-scales. J. Math. Anal. App., 328(2007), No. 2, 1217-1236.

[31] N. Sreedhar, V. V. R. R. B. Raju and Y. Narasimhulu, Existence of positive solutions for higher order boundary value problems with integral boundary conditions on time scales. J. Nonlinear Funct. Anal., 2017(2017), Article ID $5,1-13$.

[32] P . Thiramanus, and T. Jessada, Positive solutions of m-point integral boundary value problems for second-order p-Laplacian dynamic equations on time scales, Adv. Diff. Eqns., 2013(2013), Art. ID 206.

[33] S. P. Timoshenko and J. M. Gere, Theory of elastic stability, McGraw-Hill, New York(1961).

[34] S. G. Topal and A. Denk, Existence of symmetric positive solutions for a semipositone problem on time scales, Hacet. J. Math. Stat., 45(2016), No. 1, 23-31.

[35] P. A. Williams, Unifying fractional calculus with time scales [Ph.D. thesis], University of Melbourne, (2012).

[36] X. Zhang and M. Feng, W. Ge, Existence of solutions of boundary value problems with integral boundary conditions for second order impulsive integro-differential equations in Banach spaces, Comput. Appl. Math., 233(2010), 19151926.

Department of Applied Mathematics, Andhra University, Visakhapatnam, 530 003, India

*CORRESPONDING AUTHOR: rajendra92@rediffmail.com 